\title{
L'édition hors marché des ressources numérisées de la profession médicale : revisiter la fonction d'édition
}

The non-profit edition market of medical digital resources: revisiting the edition function

\section{François Horn et Thomas Lamarche}

\section{OpenEdition}

\section{Journals}

Édition électronique

URL : http://journals.openedition.org/edc/578

DOI : 10.4000/edc.578

ISSN : 2101-0366

\section{Éditeur}

Université Lille-3

\section{Édition imprimée}

Date de publication : 1 décembre 2007

Pagination : 91-109

ISBN : 978-2-9514961-8-7

ISSN : $1270-684$

\section{Référence électronique}

François Horn et Thomas Lamarche, «L'édition hors marché des ressources numérisées de la profession médicale : revisiter la fonction d'édition». Études de communication [En ligne], Numéro spécial | 2007, mis en ligne le 01 octobre 2009, consulté le 20 avril 2019. URL : http:// journals.openedition.org/edc/578; DOI : 10.4000/edc.578

Ce document a été généré automatiquement le 20 avril 2019

(c) Tous droits réservés 


\section{L'édition hors marché des ressources numérisées de la profession médicale : revisiter la fonction d'édition}

The non-profit edition market of medical digital resources: revisiting the edition function

François Horn et Thomas Lamarche

1 Parmi les développements du numérique dans la formation, un aspect singulier mérite d'être pointé. La diffusion de ressources pédagogiques par Internet est à même de fonder une nouvelle fonction d'éditeurs numériques. Dans le domaine spécifique de la médecine, le développement de l'UMVF, Université médicale virtuelle francophone, qui joue un rôle de portail de ressources pour la formation de la profession médicale, apporte quelques éclairages à cette question.

Deux caractéristiques originales et liées de cet exemple doivent être d'emblée soulignées. Premièrement, si l'on peut caractériser la fonction principale de l'UMVF comme étant celle d'un éditeur, il s'agit d'un éditeur "hors marché " dans le sens où il ne commercialise pas ses prestations. Deuxièmement, cet éditeur n'est pas issu des métiers éditoriaux mais de l'évolution d'un projet porté par un réseau de professeurs d'université en médecine dont l'objectif est de fédérer toutes les initiatives numériques médicales francophones. Ce projet, qui a connu des inflexions significatives au cours de son histoire s'est notamment appuyé sur les appels à projet Campus Numériques du gouvernement français. L'UMVF présente toutefois deux différences majeures avec les autres Campus Numériques labellisés par le Ministère. D'une part, alors que les campus numériques se sont constitués en rassemblant des équipes provenant de quelques universités partenaires, le projet de l'UMVF est de regrouper l'ensemble des facultés de médecine ${ }^{1}$ et s'est traduit par la création d'un Groupement d'Intérêt Public (GIP) auquel adhèrent 
actuellement 26 des 32 universités françaises dispensant des formations médicales. D'autre part, le projet de l'UMVF n'est pas le développement d'une offre de formation associée à un dispositif pédagogique mais la mise à disposition de ressources pédagogiques produites et utilisées par les universités partenaires. C'est en ce sens que l'UMVF a une fonction d'éditeur au service d'une profession - la profession médicale. Les spécificités de cette profession ne sont pas sans conséquence sur l'existence même de ce projet comme sur les formes qu'il a prises. C'est pourquoi, après avoir analysé ce que peut être un éditeur de contenus numérisés hors marché, nous verrons en quoi ce projet éditorial résulte de la situation singulière observable dans le champ de la médecine.

\section{Un éditeur de contenus numérisés « hors marché »?}

\section{L'UMVF un portail de ressources médicales numérisées}

3 L'UMVF se considère comme « Le portail Internet d'enseignement médical francophone »

${ }^{2}$. Les portails qui se proposent d'orienter la navigation sur le Web sont des infomédiateurs (Dang Nguyen, 1999), dans la mesure où ils jouent un rôle d'intermédiation entre les fournisseurs et les utilisateurs sur le réseau Internet. Cette intermédiation peut avoir trois origines distinctes (Brousseau et Rallet, 1999) : la communauté d'utilisateurs ou de consommateurs, des entreprises spécialisées (c'est le rôle du distributeur en général), voire le producteur lui-même qui met en forme le produit (services marketing et commercial fondant l'intermédiation). L'infomédiation peut être institutionnalisée, dans le sens où elle est organisée, reconnue et visible; elle peut aussi avoir un caractère plus informel au sein des communautés par exemple (Curien et Muet, 2004). Les portails assument formellement cette fonction de mise en marché, pas nécessairement au sens marchand du terme, mais au sens de mise en forme ou mise en scène dans l'espace public. Nombre d'entre eux dans les années 2000 sont des entreprises avec des objectifs commerciaux qui tentent de développer de nouveaux business models. Ces portails jouent le rôle de « courtier » qui orientent des demandes vers des offres (Rallet et Walkowiak, 2001). Le courtage est une fonction de traitement de données (collecte d'informations sur la nature de la demande notamment ou d'informations sur les vendeurs et les acheteurs comme dans le cas d'e-bay) dont la valeur ajoutée est essentiellement de formuler des appariements offre-demande ${ }^{3}$. Mais la traduction marchande n'est pas la seule possible, des infomédiations communautaires ou publiques coexistent. C'est le cas de l'UMVF dont la logique est délibérément non marchande.

De par son activité de portail, l'UMVF est plus un «méta campus », un réseau fédératif qu'une institution effectuant du e-learning. En effet, une activité de e-learning ne se réduit pas à une infomédiation. Certes, les apprenants se trouvent dans une situation d'utilisateur/collecteur de ressources qui nécessite une adéquation entre les fournisseurs et les utilisateurs, mais la construction d'une formation et a fortiori d'une "université virtuelle» impose de traiter, en plus de l'intermédiation, ce que l'on appelle la composante « dispositif» de formation incluant le tutorat, c'est-à-dire l'enseignement à proprement parler où les composantes relationnelles sont essentielles (Gadrey, 2003). Or ces dispositifs pédagogiques ne sont pas du ressort direct de l'UMVF.

5 Une des hypothèses principales des travaux menés dans le cadre de notre ERTE $^{4}$ est qu'une opposition forte et structurante existe entre des campus centrés sur des dispositifs et d'autres sur des ressources (Grevet, 2004). Il y a centrage sur «les 
dispositifs » lorsque les acteurs et leurs institutions d'appartenance s'engagent dans l'utilisation du numérique en partant de cursus ou de modules de formation bien précis dans lesquels sont effectivement impliqués, dès le départ, des enseignants et des apprenants. C'est pour ces cursus et ces modules que sont construits progressivement des outils et des ressources numériques, qui s'insèrent effectivement dans des dispositifs pédagogiques déterminés (Canège, Forse, CampusCultura, C@mpuSciences...). Il y a centrage sur «les ressources" lorsque les acteurs et leurs institutions d'appartenance s'engagent dans l'utilisation du numérique en privilégiant la production de ressources pédagogiques numérisées (ce fut le cas de l'UeL...) et laissent à plus tard ou à d'autres la responsabilité de l'insertion concrète de ces ressources dans les dispositifs pédagogiques des modules ou des cursus.

L'UMVF se situe clairement davantage dans l'entrée "contenus" que dans l'entrée «dispositif» au sens où elle n'est pas en elle-même une institution de formation, ne l'étant qu'à travers le réseau des universités qu'elle forme. Mais, à la différence de l'UeL, sa préoccupation n'est que secondairement la production de ressources propres. En effet, la plupart des ressources ne sont pas produites directement par le GIP-UMVF mais par les universités membres et les collèges disciplinaires. L'UMVF a donc un rôle d'éditeur même si, dans ce cas et peut-être, de façon plus générale, dans le cadre d'une édition électronique, cette fonction éditoriale comporte toute une série de particularités qui l'éloignent des définitions reconnues dans l'approche classique des industries culturelles (Miège, Pajon et Salaün, 1986).

7 Cette délimitation précise de l'activité de l'UMVF n'implique nullement une sousestimation de son rôle. Il s'agit, par la centralisation sur un même site de liens hypertextes, de donner une visibilité institutionnelle à des ressources existantes, de les coordonner et de construire une cohérence à une offre éclatée. En produisant un effet de centralisation, l'UMVF permet d'augmenter la diffusion de ces ressources et de multiplier les relations entre leurs créateurs et utilisateurs. L'UMVF effectue donc essentiellement un travail de coordination au sein d'un réseau professionnel; elle fournit un service à la collectivité éducative en médecine, et ainsi, plus largement, à la collectivité médicale, en assurant un accès unifié aux publics.

\section{Le rôle des éditeurs à l'ère de la numérisation et d'Internet}

8 Dans la société de l'information, le rôle d'éditeur occupe une place centrale dans la mesure où, pour des biens informationnels, la fonction d'infomédiation peut être assurée conjointement avec la fonction d'échange de ces biens (Curien et Muet, 2004).

Pour comprendre cette importance, il est utile de repartir des deux innovations fondamentales et interdépendantes qu'ont connues les technologies de l'information et de la communication.

La première est la numérisation potentielle de l'ensemble des contenus informationnels qui accentue la dissociation entre l'information et son support physique, aboutissement d'un mouvement très ancien de relâchement des liens entre contenus et contenants. La possibilité de reproduire et de faire circuler les données à un coût très faible, mais aussi de les modifier très facilement, bouleverse les mondes de l'édition et l'accès au savoir et à la culture. La structure de coûts des fournisseurs est profondément modifiée, mais la nature de ce qui est offert et des conditions d'usages aussi. Ainsi la migration d'une production de ressources papier vers des ressources électroniques induit de nouveaux 
coûts d'accès pour l'usager (matériel informatique et connexion, impression...) constituant de fait un transfert de coût du fournisseur vers l'utilisateur.

11 La deuxième innovation est Internet et le Web qui, en autorisant simultanément le développement des interactions personnelles et l'accès massif aux informations existantes, favorisent la construction d'un patrimoine collectif informationnel auquel chacun peut potentiellement avoir accès et contribuer.

Les fortes interactions entre ces deux innovations accélèrent leur diffusion: le développement d'Internet et $\mathrm{du}$ Web s'appuie sur la possibilité d'accéder à des informations numérisées de plus en plus nombreuses et variées. Réciproquement, la généralisation de la numérisation est stimulée par la perspective de toucher un public de plus en plus large. Il se produit un double effet d'avalanche liant développement de l'offre et de la demande (Curien et Gensollen, 1992). Les ressources numérisées accessibles par Internet occupent une place croissante et ce mouvement est appelé à se poursuivre, même s'il est évident que les ressources "papier » ne vont pas disparaitre à court ou moyen terme.

Pour ces ressources numérisées, des «éditeurs " peuvent jouer un rôle important pour deux fonctions décisives. La première concerne la production des œuvres originales, les possibilités de création collective qu'apportent les NTIC ne se réalisant pleinement que s'il existe des acteurs ayant la capacité de repérer les compétences existantes et d'organiser des collaborations fructueuses. Il s'agit là de l'édition au sens classique du mot, à savoir la coordination d'une activité éditoriale scientifique. La deuxième concerne la médiation nécessaire envers les utilisateurs qui peuvent accéder à un volume de contenus à la croissance exponentielle mais de qualité très hétérogène, et qui ont besoin pour sélectionner les contenus pertinents de repères de validation : c'est une fonction de certification de contenus, de mise en forme, de mise en visibilité, de légitimation.

Dans le domaine des connaissances scientifiques, et particulièrement en médecine, la certification des connaissances publiées, la validation des contenus, que ce soit vis-à-vis des professionnels de santé ou du grand public, sont des questions décisives. La position professionnelle d'un éditeur permet de limiter l'indétermination de la qualité, dont on sait depuis Akerlof (1970) qu'elle peut entraver le développement de l'échange. La révélation et la certification de la qualité de l'information sont en effet essentielles pour lever l'incertitude des utilisateurs, ce qui est condition d'un échange, même non marchand.

\section{Un « éditeur hors marché »}

Le domaine de la médecine semble donc en mesure de faire naître un éditeur numérique. Une singularité toutefois différencie ce modèle de "l'éditorialisation de produitservices» qui associe la commercialisation à la définition d'une activité éditoriale (Moeglin, 1998). La notion d'« éditeur hors marché » vise à caractériser un type différent d'activité en gestation, qui a des spécificités fortes concernant deux dimensions socioéconomiques importantes du modèle éditorial (Moeglin, 2005): la disponibilité et la propriété. La position continue de l'UMVF en faveur de ressources ouvertes et accessibles gratuitement à tous les publics façonne une ressource qui présente des caractéristiques d'un bien public : il n'y a pas de restriction d'accès, l'ensemble est disponible. En ce qui concerne la propriété, la logique professionnelle s'impose de la façon suivante: les producteurs de ressources ne s'approprient pas les biens informationnels dont ils sont 
auteurs (ou généralement co-auteurs). La règle est que les ressources produites le sont dans un cadre professionnel, ce qui justifie l'appropriation par l'institution. Les ressources éducatives peuvent être considérées comme un bien public produit sous l'égide des instances professionnelles.

Le fondement de ce modèle est que les ressources numérisées se rapprochent de la situation des biens collectifs purs, avec leurs caractéristiques particulières concernant leur production (indivisibilité), leur utilisation (bien non rival) et leur distribution/ acquisition (non-exclusion de l'usage). Plus précisément, les possibilités juridiques et techniques pour obtenir une contre-partie monétaire à l'utilisation de la ressource sont difficiles à mettre en œuvre et mal acceptées par les utilisateurs. C'est une des explications du fait que les éditeurs «traditionnels » n'investissent pas nécessairement l'édition numérique, à la rentabilité jugée aléatoire, surtout lorsqu'il s'agit de publications très spécialisées. Ainsi, les puissantes maisons d'édition des ouvrages de médecine ne souhaitent pas se lancer dans l'édition multimédia " où on perd de l'argent ", ne vivent pas l'arrivée de l'UMVF comme une concurrence « déloyale » et recherchent plutôt une synergie avec l'activité de l'UMVF: des accords de coopération sont en cours de finalisation portant sur la publication de livres à partir des ressources numérisées en échange d'une possibilité pour l'UMVF d'accéder aux fonds des éditeurs (papiers) deux ans après la publication des ouvrages.

Le choix d'une libre accessibilité des ressources éditées montre un certain sens de l'adaptation au développement du numérique dans la formation et a permis d'éviter les déboires qu'ont connus les tentatives de constitution de portails marchands: gestion quasi impossible des droits d'auteurs et difficultés pour financer l'activité, que ce soit par la publicité ou en faisant payer les utilisateurs, notamment à cause de la concurrence de portails, momentanément ou durablement gratuits. L'UMVF se situe dans le cas des « sites institutionnels (administrations publiques, universités [...]) dont la vocation est de fournir un service à la collectivité, et qui ayant déjà numérisé l'information qu'ils produisent [...] la mettent à disposition des Internautes » (Dang Nguyen et Pénard, 2003).

\section{Un modèle de répartition des coûts ?}

Dans la mesure où l'UMVF, au sens strict du GIP, n'organise pas les formations qui sont de la compétence exclusive des universités membres, elle n'a pas à prendre en charge le financement des dispositifs de formation et du tutorat. Elle n'est concernée que par le financement de la production et de la diffusion des ressources numérisées, c'est-à-dire de l'activité de type portail.

La décision d'inscrire cette activité dans un cadre professionnel comme un service fourni gratuitement à une communauté professionnelle, et non comme une prestation commercialisée à des clients, a évidemment des conséquences financières. Le modèle économique de la rémunération du portail ne se pose ainsi pas dans les mêmes termes que l'économie du portail marchand. Il constitue dans ce sens une forme de réponse aux nombreuses difficultés des modèles économiques du portail dans lesquels la rentabilité n'est jamais, ou difficilement, atteinte (que ce soit avec financement publicitaire ou financement payant par l'utilisateur...). Financement public et adossement à une organisation professionnelle instituée forment une voie alternative à l'économie des portails. 
20 Le caractère numérisé des ressources modifie plus la structure des coûts que leur importance. En effet, pour le producteur, la fourniture de ressources numérisées par Internet diminue radicalement les coûts de reproduction et de diffusion. Cependant, ces ressources restent marquées par un coût fixe de production de l'original, coût qui peut se révéler croissant s'il utilise les possibilités d'intégration de données de natures différentes (écrits, images, sons) et d'interaction avec les utilisateurs. Les ressources peuvent être de natures très variées, de la simple mise en ligne de documents Word à des travaux de scénarisation qui constituent une activité spécialisée coûteuse.

Pour pouvoir proposer des ressources nombreuses et de qualité, le modèle de l'UMVF repose sur trois leviers.

Premièrement, le discours de l'UMVF vise à minimiser les coûts nécessaires. Le coût de production des ressources (plus précisément le coût de transformation des connaissances que possèdent les professeurs de médecine en ressources numérisées) est considéré comme négligeable (« une fois une petite formation assurée ») ${ }^{5}$ et doit être considéré comme partie intégrante du travail «normal » de l'enseignant-chercheur. On perçoit bien la dimension performative d'une telle assertion qui vise à intégrer une activité et une compétence supplémentaires dans le travail des hospitalo-universitaires. Les coûts de production des contenus sont ainsi renvoyés à l'activité enseignante et ne sont donc pas comptabilisés.

23 Deuxièmement, les ressources étant produites par les collèges disciplinaires des différentes spécialités médicales et/ou par les facultés de médecine, ce sont les universités qui financent ces institutions qui, en fait, supportent la majeure partie des coûts.

Troisièmement, l'UMVF a néanmoins un rôle incitatif important par le soutien logistique qu'elle peut apporter (prêt des outils, des moyens de tournage...), auquel il faut ajouter plus récemment un soutien financier: les premiers succès de l'UMVF lui ont en effet permis de disposer d'un financement public plus important avec lequel elle peut contribuer au financement des projets jugés les plus intéressants.

En définitive, la compétence essentielle de l'UMVF n'est pas dans la production des ressources mais dans la mise en réseau et la visibilité. En position forte dans les institutions médicales et en situation de pionnier, le portail de l'UMVF constitue un investissement de formes au sens de Thévenot (1985), en ce qu'il produit le cadre de l'action en matière de mise à disposition de ressources numérisées. La notion de forme est ici à considérer au sens d'accès unifié à partir d'un portail unique, mais qui, à la différence là aussi de l'UeL, ne se traduit pas dans des chartes graphiques ou des homogénéisations formelles.

\section{Un modèle éditorial propre à la médecine}

Le projet de l'UMVF est de construire, dans le domaine de l'information médicale numérisée, une institution qui se présente comme étant au service de la profession médicale et qui, pour ce faire, soit représentative de celle-ci. L'UMVF assume ainsi un rôle institutionnel ou global, représentant ce que l'on nomme parfois un "acteur global» (Stockinger, 2003). La genèse du projet UMVF et les formes qu'il a prises sont profondément marquées par les particularités de la médecine. Ces caractéristiques ont 
été abondamment analysées par Freidson (1970) dans le courant de la "Sociology of the professions » (Dubar et Tripier, 1998).

\section{La profession médicale, une profession qui s'autorégule}

les universités. Les facultés de médecine ont réussi à conserver une forte autonomie au sein de l'enseignement supérieur et leurs doyens disposent d'un pouvoir particulier que l'on ne retrouve pratiquement pas ailleurs. Le terrain du numérique constituait une mise à l'épreuve de cette autonomie qui pouvait se maintenir, voire se renforcer, ou, au contraire, s'affaiblir. La création du GIP-UMVF ne s'est d'ailleurs pas faite sans tension avec les universités comme avec certains services du ministère. Par rapport aux universités, le projet de création d'une nouvelle Université Médicale, même virtuelle, pouvait susciter des craintes qui seront surmontées par la garantie que l'UMVF n'est pas et ne sera pas une université alternative organisant des formations et délivrant des diplômes, mais qu'elle est un réseau qui fédère des facultés ou des disciplines qui restent dans leur université. Par rapport au ministère, la création d'une structure centralisée fédérant le développement des différents campus médicaux rencontra l'opposition du bureau chargé de l'habilitation des campus numériques, qui, dans le domaine de la médecine, pouvait se sentir dépossédé de ses fonctions de validation, de coordination et d'attribution de financements au profit de la nouvelle entité. Mais les promoteurs de l'UMVF, dont les membres les plus actifs occupent des charges de mission au Ministère et à la Direction de la Technologie, ont montré leur capacité à trouver des appuis jusque dans les plus hautes instances décisionnelles pour vaincre les résistances et avancer régulièrement dans leur projet, y compris par l'obtention de financements substantiels. Les fortes positions institutionnelles et l'influence que peut avoir la profession médicale dans le champ de la décision politique sont également des caractéristiques marquantes de cette profession.

\section{La légitimité du monopole de la diffusion des ressources médicales numérisées}

L'UMVF, comme construction institutionnelle donnant la possibilité à la profession médicale d'autoréguler la production des ressources médicales numérisées, s'inscrit dans la tradition longue de délégation implicite par l'État de pouvoir (ou de service) public à la

Études de communication, Numéro spécial | 2007 
"communauté des médecins" ainsi en capacité de peser sur la décision publique. Le recours à la notion de mission ou de mandat (Hugues, 1958) nous permet de saisir combien la profession dans son ensemble a, et défend, une identité et un pouvoir communs. La mission publique du médecin, dont on retrouve une trace dans le discours sur l'activité de l'université dans le sens du bien public, fonde l'identité professionnelle. Il y a dans la médecine, et ainsi dans la formation en médecine, un enjeu lié à la maitrise de l'autonomie professionnelle dans le cadre de la délégation de pouvoir par l'État.

Dans le cas de l'UMVF, l'enjeu dépasse les seules questions de formation. Le rôle d'éditeur de contenus numérisés "hors marché " s'est construit et a été formalisé/théorisé progressivement. Il s'insère dans un projet plus vaste qui est à terme de fédérer tout ce qui est médical et francophone sur Internet. Ainsi, une des missions majeures que s'assigne l'UMVF (même si elle n'a pas encore débouché sur des réalisations concrètes) est de délivrer une information médicale de qualité à destination du grand public. Face à la multiplication des espaces électroniques de diffusion de savoirs médicaux et d'automédication (Demailly et Convert, 2003), l'UMVF fait figure de lieu institutionnel/ officiel. La dénomination, âprement défendue, d'« université» contribue à une reconnaissance officielle du savoir mis à disposition des publics selon un processus d'institutionnalisation d'un savoir formel (Freidson, 2001). Les enjeux en termes de formation sont largement dépassés par un enjeu de pouvoir professionnel collectif. La capacité des protagonistes de l'UMVF à être porteurs de cette mission au service de l'institution est une des principales clés de compréhension de la situation.

31 L'objectif implicite de l'UMVF est d'instaurer un monopole de la reconnaissance du savoir légitime médical numérisé. Il ne s'agit pas d'un monopole au sens juridique, mais d'une forme de monopole de l'accès aux connaissances médicales électroniques reconnues comme telles par la profession et construites par le biais d'une offre unifiée et d'un accès commun. Ce projet est en parfaite adéquation avec la position de monopoles multiples qui caractérise la profession médicale. Le monopole sur le marché des soins est le plus fondamental : c'est la « revendication morale du monopole d'exercice » (Dubar et Tripier, 1998) qui a permis le succès de la médecine moderne face aux savoirs ancestraux. Le monopole (ou le contrôle) sur le marché du travail donne à la profession ses propres clés d'accès. C'est là une des composantes essentielles du "professionnalisme » au sens de Freidson: l'Université a le monopole de fourniture des autorisations d'accès à la profession en délivrant la «licence » au sens de Hugues (1958), c'est-à-dire l'autorisation d'exercer. D'où l'importance du contrôle de la formation universitaire et le rôle particulier des hospitalo-universitaires qui reçoivent de l'État un double mandat, en tant que médecin et en tant qu'enseignant, et dont on peut considérer qu'ils relèvent ainsi d'un double « professionnalisme ».

La principale difficulté était de faire reconnaitre la légitimité de l'UMVF à exercer ce rôle tant par rapport aux disciplines médicales (et à leurs institutions existantes) et aux facultés de médecine que par rapport aux ministères (santé et surtout éducation).

Par rapport à l'État, le projet de favoriser le développement et la visibilité internationale de ressources numérisées francophones et libres d'accès peut apparaître comme un moyen efficace de défense de la mission de l'université de médecine française face à la «pression » d'universités étrangères développant l'enseignement à distance. A l'opposé d'une vision corporatiste, la démarche se présente comme soucieuse de l'intérêt général. La gratuité d'accès aux ressources répond implicitement à la menace de la commercialisation des services qui prend place dans le cadre des négociations de l'AGCS 
(Lamarche, 2003). La ténacité des porteurs du projet pour défendre cette orientation est d'autant plus remarquable qu'elle se situe dans le milieu de la formation médicale où des prestations marchandes complémentaires de l'enseignement public occupent une place beaucoup plus importante que dans les autres formations, le recours à ces prestations étant quasiment indispensable pour la réussite des études.

Par rapport à la profession médicale, la construction de la légitimité de l'UMVF résulte de sa capacité à produire un discours au nom de la profession. La production d'un savoir comme bien public joue un rôle d'unification et de construction symbolique. On voit donc toute l'importance d'une institution commune à la profession, pour contrôler l'accès aux savoirs, aux marchés du travail et au final à la profession. La justification professionnelle commune recouvre une constellation de collèges représentant chaque spécialité mais le discours politique/général fait ordre et fait raison auprès des composantes du monde hospitalo-universitaire. La défense d'une sorte d'intérêt général de la communauté médicale devient ainsi un moyen très puissant de transcender les conflits, distances et oppositions entre les corps, les spécialités et les universités qui la composent. Toutefois, derrière ce "monopole " d'accès aux savoirs officiels l'ensemble des collèges et des universités reste très libre.

\section{Des formes de structuration originales}

La structuration de l'UMVF ne repose pas sur des logiques de marché ; il n'y a en effet pas de transaction, l'essentiel est gratuit et la perspective n'est pas de construire des prestations commercialisables. Elle ne repose pas non plus sur un ordre «bureaucratique ». Le pouvoir et les décisions ne passent pas par l'élaboration de règle formelle, de norme ou de contrainte ; la mise en réseau des acteurs universitaires n'a pas été rendue obligatoire, ni normée par une autorité. Ainsi la coordination relève d'une forme de coopération au sein d'un monde professionnel.

Dans certains cas, la numérisation et Internet ont permis l'émergence de nouvelles formes organisationnelles, constituées de communautés de pairs (Gensollen, 2004). L'architecture distribuée qui caractérise Internet (Curien et Muet, 2004) peut en effet bouleverser les hiérarchies existantes par le développement de relations essentiellement horizontales. Par contraste, la coordination par le réseau est ici atypique. L'architecture du vaste ensemble qu'englobe l'UMVF forme une hiérarchie, une verticalité qui s'oppose à différentes coordinations professionnelles qui sont au contraire basées sur des formes d'horizontalité (Delamotte, 2004).

Insérés dans les rouages de l'ordre médical, les porteurs du projet parviennent à imposer un type de campus centralisant les ressources, qui est plus un modèle descendant vers les usagers qu'ascendant depuis ceux-ci (Bon et Louppe, 1980). Sous réserve d'une analyse plus approfondie des usages, il semble ainsi que le réseau soit plus au service de la profession que des étudiants usagers. En effet, alors que la question de la construction des liens médiatés ${ }^{6}$ est une importante question dans l'économie d'Internet (Gensollen, 2004, Ngo-Mai et Raybaut, 2004), la situation des ressources numériques dans l'enseignement médical est particulière : une réponse est apportée sur le versant de la fourniture de ressources sans considération particulière sur l'appariement de l'offre à la demande. Caractéristique des organisations descendantes, une offre centralisée se réalise sans attention particulière aux pratiques effectives. 
38 L'UMVF s'inscrit donc dans la continuité d'un modèle professionnaliste par opposition au modèle industrialiste (Grevet, 2005). Mais sa structuration est le reflet d'une profession médicale qui a un ordonnancement et une hiérarchie très marqués. Le projet de l'UMVF, impulsé et coordonné " par le haut », correspond à un monde professionnel comprenant des fortes relations d'ordre au sein du monde de référence, ici le monde hospitalouniversitaire. Ainsi l'UMVF peut être caractérisée comme constituant une forme originale de « communauté professionnelle encadrée ».

\section{Conclusion}

39 Le domaine médical nous permet de saisir la trajectoire de constitution d'un «éditeur hors marché », trajectoire qui repose sur une double justification, institutionnelle et professionnelle. La mise en place d'un portail rendant accessible et certifiant les ressources pédagogiques, sans toutefois les produire directement, correspond à une fonction éditoriale.

L'UMVF a probablement atteint un seuil pour s'instituer comme le portail de la profession hospitalo-universitaire francophone. Les apports de l'économie des réseaux permettent de comprendre comment se développent des processus cumulatifs d'adoption et l'existence de situation de type « winner takes all » dans lesquelles l'avantage de départ est si fort qu'il réduit pratiquement à néant toute initiative rivale ${ }^{7}$. A partir de l'existence de rendements croissants d'adoption, Brian Arthur (1988) a mis en évidence les mécanismes "d'autorenforcement » qui se créent autour d'une technologie : l'action même d'adopter une technologie rend celle-ci plus attractive pour les utilisateurs potentiels, augmentant par-là même ses chances d'être adoptée dans le futur. Se produit un effet feedback positif (Shapiro et Varian, 1999) qui caractérise le ralliement des différents acteurs (dans la situation observée, il s'agit des collèges disciplinaires et des facultés). En rendant leurs ressources accessibles via ce portail, ces acteurs contribuent à construire la crédibilité de l'UMVF, ce qui favorise l'agrégation de nouveaux acteurs réticents au départ par rapport à cette initiative. Il en résulte un renforcement de la position institutionnelle de l'UMVF qui lui permet de bénéficier de sources supplémentaires de financement, qu'elle peut redistribuer pour contribuer à la production de nouvelles ressources augmentant la quantité et la qualité de l'information médicale numérisée disponible.

Deux questions restent ouvertes cependant.

42 La première concerne la généralisation possible de cette expérience hors de la médecine. L'UMVF se considère, et est considérée par le ministère, comme la préfiguration des Universités Numériques Thématiques (UNT) nationales et comme un modèle pour les autres disciplines. Le ministère affiche ainsi sa volonté de constituer dans tous les domaines de l'enseignement supérieur des UNT en suivant l'exemple de la médecine et le mouvement de création des UNT gagne de nouvelles spécialités (droit, gestion, sciences...). Il nous semble toutefois que, tant le projet d'édition numérique de l'UMVF que ses modalités pratiques de mise en application dépendent étroitement du cadre institutionnel de la profession médicale. Identifier précisément ce qui relève des spécificités de cette profession peut permettre d'éviter des désillusions dans les tentatives de transposer mécaniquement le modèle à d'autres professions ou disciplines. Sans verser dans une vision idiosyncrasique, il est nécessaire de considérer les facteurs instituants en place dans la médecine et dans les autres spécialités thématiques candidates aux UNT. Le 
modèle d'éditeur hors marché que l'on peut repérer pour la médecine est, nous l'avons montré, très fortement marqué par le monopole qu'exerce la profession médicale, monopole qui est facteur d'unité. Or, la plupart des spécialités thématiques candidates aux UNT sont le fait d'une pluralité d'acteurs et sont notamment marquées par des formes de coexistence, voire de concurrence en matière d'offre de formation (la rivalité grandes écoles - université étant la forme la plus évidente) qui rend plus problématique la question de la certification des ressources pédagogiques. La question que l'on peut se poser n'est pas celle de savoir s'il y aura des UNT, car il peut y avoir une construction institutionnelle supplémentaire portée par le ministère, mais celle de savoir comment cet "étage " institué au niveau de la discipline ou de la spécialité peut s'imposer comme légitime vis à vis de la profession (en l'occurrence les producteurs de ressources pédagogiques). Si dans le domaine de la médecine l'histoire a fondé une logique d'ordre qui reste prégnante, la plupart des autres spécialités restent elles à l'inverse marquées par des logiques de rivalités. Dès lors, le modèle éditorial unifié que nous avons tenté de décrire est difficilement reproductible car il ne peut pas reposer sur le "monopole " exercé dans la validation des savoirs et ressources mis en public. Le poids des formes de la concurrence est en fin de compte fortement structurant et rien ne permet de penser que les UNT ne seront pas des éditeurs de ressources; en revanche, il est difficile d'imaginer qu'elles puissent atteindre la même puissance institutionnelle au sein de leur spécialité.

La deuxième question porte sur l'utilisation réelle des ressources éditées par des personnes autres que celles qui les ont développées. Un modèle de diffusion libre des ressources qui s'inscrit dans la tradition d'un service public d'enseignement peut a priori susciter des interrogations sur le fait que les ressources mises en ligne répondent effectivement à des usages avérés, sur l'adaptation fine des ressources aux usages existants et sur leur appropriation réelle par les enseignants dans leur pratique professionnelle et par les étudiants dans leur formation. De par son caractère nonmarchand, l'UMVF n'est pas soumise à une contrainte économique d'atteindre des objectifs de fréquentation et d'utilisation de ses ressources et il importe de recourir à d'autres indicateurs que le marché pour mesurer l'adaptation effective des ressources éditées aux besoins des utilisateurs. Mais, pour l'instant, il ne semble pas que le souci d'étude sur les usages effectifs ait beaucoup préoccupé les acteurs du projet.

\section{BIBLIOGRAPHIE}

Akerlof, G., (1970), « The market for lemons. Qualitative unicertainty and the market mechanism », Quaterly journal of economics 84, 488-500.

Andreff, W. et Szymanski, S., (éds.), (2006), The Handbook on the Economics of Sport, Edward Elgar, Cheltenham.

Arthur, B., (1988), « Competing Technologies : An Overview », in : Dosi G., Technical change and economic theory, Pinter.

Benhamou, F., (2002), L'économie du star system, Odile Jabob, Paris. 
Bon, J. et Louppe, A., (1980), Marketing des services publics. L'étude des besoins de la population, Les éditions d'organisation, Paris.

Brousseau, E. et Rallet, A., (1999), Technologies de l'information et de la communication, organisation et performances économiques, Commissariat Général au plan, Paris.

Curien, N. et Gensollen, M., (1992), Économie des télécommunications, ENSPTT-Economica, Paris.

Curien, N. et Muet, P.-A., (2004), La société de l'information, La documentation française, Paris.

Dang Nguyen, G., (1999), « Du commerce électronique à l'intermédiation électronique », in : Brousseau E. et Rallet A., Technologies de l'information et de la communication, organisation et performances économiques, Commissariat Général au plan, Paris.

Dang Nguyen, G. et Pénard, T., (2003), Marchand et non-marchand sur internet : rivalité ou complémentarité ?, École d'été du GDR TIC et société (http://perso.univ-rennes1.fr/ thierry.penard/biblio/DangPenardcarry.pdf).

Delamotte, E. (éd.), (2004), Du partage au marché. Regards croisés sur la circulation des savoirs, Septentrion, Lille.

Demailly, L. et Convert, B., (2003), « Le savoir médical et sa mise en scène sur le net », Terminal $89,67-76$.

Dubar, C. et Tripier, P., (1998), Sociologie des professions, Armand Colin, Paris.

ERTe CN, (2005), Résultats 2005 de l'ERTe (Équipe de Recherche Technologique éducation) " Modèles économiques et enjeux organisationnels des campus numériques », résultats accessibles à www.ifresi.univ-lille1.fr/SITE/2_Recherche/22_Programmes/ERTe/ERTe.htm ou à partir de la page d'accueil www.mshparisnord.org/.

Freidson, E., (1970), Professional Dominance : The Social Structure of Medical Care, Atherton, New York.

Freidson, E., (2001), Professionalism, the third logic. On the practice of knowledge, University of Chicago presse, Chicago.

Gadrey, J., (2003), Socio-économie des services, La découverte, Paris.

Gensollen, M., (2004), «Biens informationnels et communautés médiatées », Revue d'économie politique (http://www.enst.fr/egsh/enstcommed/workinprog.htm) 113, 9-40.

Grevet, P., (2005), « L'expérience économico-institutionnelle de Canége », accessible aux adresses indiquées à ERTe CN 2005.

Grevet, P., (2004), « Note à la suite du rapport d'expertise et du travail déjà engagé dans l'ERTe ».

Horn, F. et Lamarche, T., (2005), « L'Université Médicale Virtuelle Francophone (UMVF) », accessible aux adresses indiquées à ERTe CN 2005.

Hugues, E. C., (1958), Men and their work, The Free Press, Glencoe.

Lamarche, T., (2003), «L'OMC et l'éducation : Normalisation de l'éducation pour en faire une marchandise échangeable internationalement? ", Politiques et management public 21, 109-130.

Miège, B., Pajon, P. et Salaün, J.-M., (1986), L'industrialisation de l'audiovisuel. Des programmes pour les nouveaux médias, Aubier, Paris.

Moeglin, P., (1998), L'industrialisation de la formation. État de la question, CNDP, Paris.

Moeglin, P., (2005), Outils et médias éducatifs, PUG, Grenoble. 
Ngo-Mai, S. et Raybaut, A., (2004), « Communautés de consommateurs et marché infomédié », Revue d'économie politique 113.

Petit, P., (2006), « Formes structurelles et régime de croissance de l'après fordisme », Working paper Recherche et Régulation (www.theorie-regulation.org).

Rallet, A. et Walkowiak, E., (2001), Économie de l'Internet et localisation des emplois : l'exemple des services d'intermédiation sur Internet, $37^{\mathrm{e}}$ colloque de l'association de science régionale de langue française, Bordeaux.

Shapiro, C. et Varian, H., (1999), Économie de l'information : Guide stratégique de l'économie des réseaux, De Boeck Université, Paris.

Stockinger, P., (éd.), (2003), Portails et collaboratoires pour la recherche et l'enseignement, Lavoisier, Paris.

Thévenot, L., (1985), « Les investissements de formes », Cahiers du Centre d'études de l'Emploi 29, 21-71.

\section{NOTES}

1. Bien que supprimée depuis 1969, l'appellation "faculté " reste présente dans le domaine médical (comme dans les filières de droit), ceci dans le langage courant, dans les communications, voire dans certains documents officiels des universités ou des UFR sans avoir de fondement légal. Nous reprenons cet usage qui nous semble exemplaire de pratiques visant à la construction de l'autonomie au sein de l'université.

2. C'était le titre de la page d'accueil du site de l'UMVF, qui a été remplacé au cours de l'année 2004 par un plus modeste «Portail Internet de l'Université Médicale Virtuelle Francophone ».

3. Voir aussi dans ce même numéro l'article de P. Moeglin.

4. Résultats disponibles (ERTe CN, 2005).

5. Selon la formule d'un des porteurs de l'UMVF (cf. Horn et Lamarche, 2005).

6. Le lien entre les internautes n'est pas médiatisé par Internet selon Michel Gensollen qui propose la notion d'économie médiatée pour caractériser les relations des communautés d'internautes. "Ces 'communautés médiatées' sont des structures d'interaction originales: elles se caractérisent par une quasi-absence de lien social interpersonnel et par la construction et l'utilisation solitaires d'un objet informationnel commun » (Gensollen, 2004).

7. Pour des exemples de situation où le "vainqueur emporte tout ", cf. dans l'économie du spectacle : Benhamou (2002), et dans l'économie du sport : Andreff et Szymanski (2006).

\section{RÉSUMÉS}

La médecine et son projet d'Université Médicale Virtuelle Francophone permet d'analyser la tentative de constitution d'un "éditeur hors marché ", démarche qui mobilise une double justification institutionnelle et professionnelle. La création d'un portail permettant l'accès à des ressources, sans les produire directement, correspond à une fonction éditoriale qui est 
transformée par la numérisation et Internet et dans laquelle le rôle de certification des ressources est essentiel.

Un modèle éditorial original se met en place progressivement, fortement marqué par les spécificités de la profession médicale, notamment le monopole qu'exerce l'ordre médical dans la régulation du champ professionnel. En conséquence, sa généralisation indépendamment de son succès - et de ses limites - apparaît délicate.

The French medical field and its Virtual Francophone Medical University (Université Médicale Virtuelle Francophone) project give us the occasion to analyse the construction process of a for "non-profit editor». This process used two justifications: institutional and professional. Creating a portal that aggregates resources that its owners did not produce seems to be a new editorial function derived from digitization and the Internet where certification of resources is an essential role.

An original editorial model is appearing. Its current success is profoundly linked to the specificities of the medical profession. In particular to the monopoly that the medical order exerts over regulations in the medical field. But this renders difficult the generalisation of the experience to other sectors, despite of its success - and limits.

\section{INDEX}

Keywords : virtual publishing, portal, profession, medecine, digitisation, cost, ressource

Mots-clés : édition numérique, portail, profession, médecine, numérisation, coût, ressource

\section{AUTEURS}

\section{FRANÇOIS HORN}

François Horn est maître de Conférences en Sciences économiques à l'université Charles-deGaulle - Lille 3. Ses recherches effectuées dans le cadre du CLERSE-IFRESI (CNRS) portent sur l'économie des nouvelles technologies de l'information et de la communication. Elles concernent l'économie des logiciels (cf. notamment L'économie des logiciels, La Découverte, Repères $n^{\circ} 381$, 2004) et plus généralement les nouvelles formes de production, de circulation et d'usage des informations à l'ère de la numérisation et d'Internet. Adresse électronique : francois.horn@univlille3.fr.

\section{THOMAS LAMARCHE}

Thomas Lamarche, économiste, est membre du Germe de l'Université Paris 7. Il mène des recherches interdisciplinaires associé à GERIICO, Université Lille 3. Deux orientations de recherche : l'une sur la marchandisation des services publics et particulièrement de l'éducation (en lien avec la mondialisation et le développement du e-learning, $c f$. Éducation et capitalisme (éd.), Syllepe-Nouveaux regards, 2006), l'autre sur la responsabilité sociale des entreprises en inspectant sa dimension communicationnelle mais aussi la recomposition du capitalisme qu'elle recèle ( $c f$. avec $\mathrm{P}$. de la Broise, 2006, Responsabilité sociale : vers une nouvelle communication des entreprises?, Septentrion). Adresse électronique : thomas.lamarche@univ-lille3.fr. 